Research Article

\title{
Criterial Based Opinion Leader's Selection for Decision-Making Using Ant Colony Optimization
}

\author{
Xu Qiang $\mathbb{D},{ }^{1}$ Zhao Huiqi $\left(\mathbb{D},{ }^{2}\right.$ Farhad Ali $\mathbb{D},{ }^{3}$ and Shah Nazir $\mathbb{D}^{3}$ \\ ${ }^{1}$ College of Computer Science and Engineering, Shandong University of Science and Technology, Qingdao, Shandong, China \\ ${ }^{2}$ College of Intelligent Equipment, Shandong University of Science and Technology, Tai'an, Shandong, China \\ ${ }^{3}$ Department of Computer Science, University of Swabi, Khyber Pakhtunkhwa, Pakistan
}

Correspondence should be addressed to Xu Qiang; myxinxi@sina.com and Shah Nazir; snshahnzr@gmail.com

Received 26 May 2021; Accepted 8 July 2021; Published 28 July 2021

Academic Editor: Zhu Xiao

Copyright (c) $2021 \mathrm{Xu}$ Qiang et al. This is an open access article distributed under the Creative Commons Attribution License, which permits unrestricted use, distribution, and reproduction in any medium, provided the original work is properly cited.

The Internet has already become a medium for people to access information, express their views, and connect with one another through various media and tools as a result of its rapid global development. Web 2.0 has become increasingly important in people's daily lives. With the passing of time, many online communities, such as blogs, forums, and websites, are becoming more interactive. Individuals can easily express their opinions publicly and see what others say about their opinions. In a social network, an opinion leader is a powerful individual who is an expert in a particular field and has a large number of people who follow his or her comments or ideas. Companies and governments may contact the most powerful people after discovering them in order to influence sales or guide public opinion, respectively. In heterogeneous communication networks, opinion leaders serve as an influencer for the acceptance and dissemination of new products and users in marketing. Others' drives, values, perceptions, motivations, and actions can be influenced by opinion leaders. These influences have a major effect on other customers' policymaking processes. Opinion leaders come in a variety of demographic shapes; in general, they are committed, ambitious, and dynamic individuals with excellent academic and social edifications who persuade customers through their expert knowledge. Opinions and values are the most important variables in human experiences in social phenomena. The method of assessing opinions is extremely difficult since people often change their minds when speaking with others. The study of the evolution and formation of opinions in a social group is known as opinion dynamics, and it is based on collective decision-making. The bounded confidence rule is one of the intrinsic interaction principles in the dynamics of human actions. Many models have been created to date to explore the dynamics of leaders' decision-making. Due to the social network's high complexity and the randomness and contingency of their behavior, identifying opinion leaders in the network is difficult. Organizations and entrepreneurs must choose which opinion leaders to partner with to gain a deeper understanding of how to communicate with them to promote various goods and services. For the opinion leader's selection optimization, the proposed framework feature-based opinion leader selection is utilized using the ant colony optimization technique that selects the best opinion leader based on their unique features. The proposed method will address the opinion leader selection problem.

\section{Introduction}

With the Internet's rapid global expansion, it has become a means for people to access information, express their opinions, and interact with one another through a variety of media and resources. After newspapers, radio, and television, the network is considered the "fourth media," and it has evolved into one of the most powerful platforms for expressing social public opinion. Social networking has given birth to a gigantic virtual community, and with the development of networking technologies, there is an increased interest in utilizing public opinion [1]. Virtual forums and networking platforms have expanded people's conventional social settings and personal learning networks. People's lives have been transformed as a result of online communication, which has aided knowledge sharing, learning, and the dissemination of useful information [2]. Web 2.0 has become increasingly important in the public's 
daily lives. Many virtual societies, such as blogs, forums, and websites, are becoming more interactive. People can quickly express themselves and read what others have to say. An opinion leader is a powerful person who is a specialist in a particular field and has a huge following in a social network. Opinion leaders, without a doubt, are wisdom creators and message senders who have a working knowledge of the media by secondary transmission. The economic and political consequences of identifying opinion leaders in a social network are important. Further to their identification, businesses and governments can contact the most powerful people in order to influence sales or influence public opinion. Researchers can find out where public opinion comes from by detecting the most common tweets [3]. In heterogeneous communication networks, opinion leaders serve as influencers for the acceptance and dissemination of new products and users in marketing. Others' drives, values, perceptions, motivations, and actions can be influenced by opinion leaders. They can be pronounced as illustrating influencers of word of mouth. These influences have a major effect on other customers' policymaking processes. Opinion leaders come in a variety of demographic shapes; in general, they are committed, ambitious, and dynamic individuals with excellent academic and social edifications who persuade customers through their expert knowledge [4].

A common application of web 2.0 is a social networking site (SNS). Users can exchange ideas, obtain knowledge, and communicate emotionally on social media sites. People's everyday lives are influenced by social media. Opinion leaders are unique consumers of social media platforms, with higher advanced degrees and a wider following. The views of opinion leaders also affect the public opinion of nearby users and the entire network. Opinion leaders, obviously, play a critical role in social media [5]. Opinion leaders in virtual communities play a significant role in influencing public opinion, and their superior status, leadership, and social influence attract many followers [2]. The word "public opinion" refers to a social space that encompasses the occurrence and creation of intermediate social events and changes, as well as the general public as a subject of social management, corporations, individuals, and other types of organizations, and their political, social, and moral orientations and social attitudes. It is the sum of many people's thoughts, perceptions, emotions, and feelings about social issues and phenomena. Network public opinion discusses how netizens use the Internet to express and share a wide range of feelings, attitudes, and viewpoints. Text, photos, audio, and video distribution and transmission, which is mainly in written form, are used to communicate and distribute these feelings, attitudes, and viewpoints through the network. Two characteristics of network public opinion are interactivity and ontology timeliness, as well as the following: a strong rational emotional personal culture emerges from the shadows with controllable equality interactivity [1].

Through the advancement of communication technology, the Internet is now used not only to improve information transfer, increase people's efficiency and productivity, and promote social interaction, but also to make it possible to participate in the process of social events because of its characteristics such as anonymity, instantaneous engagement, and nonintermediary, which have burst through a barrier. Furthermore, the poor in reality's personal views will coalesce on the Internet, influencing or even challenging the official popular consciousness. This kind of continuity resulted in NPO. It can be described as a consistent point of view resulting from the enhancement of social events and, in particular, Network transfers [6]. The most critical factors of human interactions in social environments are opinions and beliefs. Since people often change their minds when interacting with others, assessing opinions is extremely difficult. The study of collective opinion change through interactions among agents is emphasised in opinion dynamics. The Hegselmann-Krause (HK) design is responsible for the opinion dynamics model, which is now one of the most extensively researched methods in this area. Bounded confidence models, such as the Hong Kong model, are a form of bounded confidence model. Only when the difference in their opinions is less than a certain bounded confidence level does a pair of agents communicate in the $\mathrm{HK}$ model. Agents in the original HK model have a common set of characteristics such as level of trust, knowledge, and their impacts on other agents. In the real world special agents have a stronger influence over other agents [7].

Opinion dynamics is the progression and development of opinions in a societal community; open dynamics is based on decision-making. One of the intrinsic interaction concepts in the dynamics of human behavior is the bounded confidence law. When in a social group no opinion leader is present, the initial views, trust levels, and group size will determine the progression of the group's mutual opinion in bounded confidence-based opinion dynamics. Almost every aspect of a person's life in a social group is influenced by their beliefs and opinions. A complex social network is formed when social agents interact with one another. Opinion dynamics explains how mutual views shift as a result of a network's composition [8]. Many models were developed to study the dynamics of leaders to date. However, due to the social network's high complexity and the randomness and contingency of their acts, identifying opinion leaders in the network is difficult. Opinion leaders are important in the initial market development and successful diffusion of new (especially high-tech) products and services. Opinion leaders have been observed to have both an informal and formal impact on the distribution and acceptance of new goods, as well as to influence other people's choices via different communication networks. As a result, companies are increasingly interested in opinion leaders, and they are now considered a valuable first marketing target [9]. Organizations and entrepreneurs must choose which opinion leaders to partner with to gain a deeper understanding of how to communicate with them to promote various goods and services. Several approaches have been suggested to improve the selection of opinion leaders but none of them focused on the selection of opinion leaders based on their unique features. For the opinion leader's selection optimization, the proposed framework feature- 
based opinion leader selection is utilized using ant colony optimization technique. The proposed method will address the opinion leader selection problem.

\section{Literature Review}

Network public opinion is becoming a significant social theory as the Internet grows in popularity and information network technology advances. Based on existing research, two forms of spreading were proposed: internal and external. The two modes of NPO are evolution and diffusion. Opinion holders, as special agents in the spread of NPO, often hold a role on social issues, which affects both the ideas of the whole party and the spreading process. Some models were developed based on the modelling ideas of the Weisbuch-Deffuant model, while also taking into account the characteristics of network communications and the special circumstances of NPO, to better understand how opinion leaders influence NPO dynamics. Experiments show that the involvement of opinion leaders has a major impact on NPO polarization, and even determines it, particularly when the opinion leaders' ideas are specious. The NPO diffusion method has developed a complicated information diffusion network because the operators are not independent of one another. SIR models were used to study the effects of different network topology structures on the diffusion process in this case. When a random network with a rich-club effect is compared to the same network node, edge, and mean degree, the result shows that NPO not only diffuses faster but also occupies a higher rate of the impacted agent [6]. It was anticipated as a social society with several subgroups of influential people. When another two opinion leader demographic groups held opposing target views, a fuzzy HK model for opinion followers and leaders was formed. Because it is more applicable to real-life situations, the familiarity of opinion leaders' views is used to determine their influence over their followers. Some computer simulation results based on the proposed fuzzy opinion dynamic model were generated to validate the new model and investigate the impact of different opinion distance and weight segmentation methods on the process of the followers' opinion evolution. According to the simulation results, when weight or opinion gap memberships are partitioned more deeply, followers are more highly influenced by leaders. The results show that the proposed model works well and is consistent with previous theoretical findings in the field of opinion dynamics [7].

The delimited confidence concept was used to create a model for opinion dynamics sponsored grouping of radical participants. The majority of the party's leaders were divided into three groups: opinion supporters, positive opinion leaders, and divisive opinion leaders. In order to test the current model, some simulation findings based on the predicted opinion dynamics model are discussed, as well as the effects of opinion leaders and trust levels on opinion following. According to the simulation results, confident leaders have a stronger influence on their supporters as their confidence in them increases. Simultaneously, as followers' confidence grows, the leaders' power grows, and followers are more likely to reach an agreement. The study's results revealed that in public administration, a governmental organization's public integrity is crucial, and that cautious measures must be formulated to align leader control with member confidence levels [8]. Mining opinion leaders in a large social group is difficult due to difficulties with graph analysis and leadership assessment. OLMiner is a unique collection of rules that combines group structure and leadership content analysis techniques to find opinion leaders in a large social community. The effect of the influence overlapping hassle in social networks was considerably decreased using two-level clustering techniques. Sentiment analysis is regularly utilized by OLMiner to decide the opinion sample of determined opinion leaders. Lastly, to make certain of its feasibility, the proposed set of rules was checked on actual datasets. The experiments show that, with the use of a variety of parameters, OLMiner is able to detect more knowledgeable opinion leaders and correctly resolve the hassle of overlapping power [3]. To detect opinion leader nodes in online social networks, the relationships among opinion leaders and topological traits of nodes are revealed [1].

Based on communication theory, a technique for designing social networks based on communication probability was suggested. Based on the traits of opinion leaders in phrases of professionalism, strength, and interaction, a corresponding index system was proposed. Following that, a measurement model focusing on social group studies is introduced. The results suggest that opinion leaders are a small organization in society that requires continuous interaction with others in order to rise in power. Similarly, the PCA-SNA model can be used to categorise opinion leaders, which has consequences for SNS implementation [5]. For LSGDM problems, a k-core decomposition-based opinion leader identification system and a clustering-based consensus model were created. To begin, a similarity-based clustering technique is proposed for grouping decision-makers (DMs) into multiple clusters. Subclusters that are made from DMs with equal alternative rating information are then presented for the development of social networks (SNs) [10]. A new approach for measuring cloud trust was proposed using opinion leaders and troll organizations. Trust mechanisms of cloud systems were also investigated. The trust ranking in the cloud is measured using certificate attributes such as affordability, security, data quality, and identity, as well as trolls and opinion makers. Unlike other methods, the new scheme takes into account negative correct opinions, negative incorrect opinions, positive correct opinions, and positive incorrect opinions. According to the results of dataset experiments, the system outperforms other approaches. Furthermore, the proportion of opinion makers and troll bodies was discovered to be equal to their reputation. As the reputation rating is greatly increased, fewer opinion figures are chosen, yet more troll entities are discovered. It is possible to obtain a clearer meaning for credibility and trust by eliminating the power of troll entities by using the advice of opinion makers. The accuracy of the proposed procedure was seen to have improved [11].

For a community of social actors, the bounded confidence rule was used to create a leader-follower opinion forming model. Opinion leaders and opinion supporters 
were separated from the rest of the party based on their differing levels of confidence. Environmental noises were factored into the opinion dynamics to model environmental uncertainty. To test the current model and investigate the effect of opinion leaders and ambient noises on opinion followers, several computer simulation findings based on the proposed opinion formulation model were created. When the group size and number of leaders are set, the evolution of the followers' views under various environmental noises is perceived. According to the simulation data, when ambient noise variances exceed a certain level, opinion leaders have a significant effect on followers. As the sounds become louder, the leaders' influence over the followers dwindles. According to the simulation results, as the fraction of opinion leaders grows, the impacted fraction of followers grows until the fraction of opinion leaders hits the optimal fraction. As the quantity of opinion leaders approaches the optimal fraction, the cumulative influence of opinion leaders on followers deteriorates. The results confirmed the proposed leaderfollower opinion dynamics model's efficiency, as well as recent theoretical research on decision-making and opinion distribution in real civilizations [12]. Using multiple opinion leader search algorithms at the same time allows you to filter out users who are not likely to become potential opinion leaders. This could save time and money when it comes to network analysis. As a result of the research, it was suggested that not only the user's position in a social network but also parameters of his actions when interacting with other users be considered. When the proposed algorithm is compared to other algorithms, it is discovered that all of them regard certain nodes as potential leaders. However, since the suggested algorithm is based on user accuracy, certain parameters can only be considered while using it. This may be particularly useful when discussing users who, based on the nature of their activity, may become more relevant in the near future. As a result, this algorithm makes it possible to find dependencies that would otherwise be difficult to find based solely on a user's network location and the number of his neighbors [13].

The author proposes a framework for detecting opinion communities that takes into account information, time, and user topology structure. It is possible for two users to have integrated similarities in terms of material, time, and topology structure. The opinion groups are then identified based on their shared similarity. In addition, based on usage effects and emotional insight, a tool for identifying opinion leaders is proposed. The opinion community is made up of users who share similar preferences. Meanwhile, a directed graph is used to represent the relationship between opinion community users. The user impact model and the emotional analysis model were then investigated. The emotional analysis model frequently takes into account the intensity of negative expressions. The impact worth model is then developed for each customer in the opinion community. Finally, the power of the proposed algorithms is determined in a distributed computing environment. In the meantime, extensive research is being carried out. The results show that our proposed opinion population detection algorithm can accurately identify opinion groups. Furthermore, the proposed algorithm for identifying opinion leaders in social networks has high accuracy in recognizing opinion leaders [14]. A modern Social Network-based Whale Optimization Algorithm (SNWOA) was used to detect the Top-N opinion leaders by measuring the user's credibility in the network using various mathematical optimization functions. Because it is based on humpback whales' bubble-net hunting activity, the proposed algorithm is useful for identifying opinion leaders. As the number of users in the network grows, the algorithm seeks the best solution possible; thus, the algorithm's overall complexity remains unaffected. A new approach for classifying communities has been proposed which makes use of a similarity index that includes neighbor similarity and clustering coefficients. After measuring each user's output index using their centralities, the proposed approach was used to identify local and universal opinion leaders using different optimization functions. The conceptual algorithm was put to the test on real and synthetic datasets, with accuracy, precision, recall, and F1-score used to compare the results. The proposed algorithms outperform the other conventional Social Network Analysis (SNA) measures, according to the findings. Other population detection algorithms that are based on different parameters and computational time outperform the group partitioning algorithm [15].

A brand new structure for identifying opinion leaders is proposed based on customer trust interactions. Recognized opinion leaders can be used on a variety of subjects, including politics, economics, education, and social issues. After filtering the values of datasets in three parts (removing self-confidence remarks, duplicate comments, and troll comments), the proposed method employs parts: trust evaluating and opinion leader selection to identify customers with the highest ordinary trust value. The JC metric, which is based on structural and social similarities between customers, is used to assess the strength of trust relationships. As a consequence, in this analysis, opinion leaders are characterised by calculating the overall confidence value from customer feedback [16]. To find the alliance of opinion leaders based on the group's optimal mutual synergy, a game theory-based strategy was used. Three forms of trust were also suggested for determining a user's level of trust. The Shapley value is often used to calculate a coalition's average marginal contribution, which is then used to produce synergy. To precisely classify the opinion leaders in a social network, a consumer most likely communicates with others using degree networks, game theory logic, confidence, and conditional probability. In comparison to other SNA strategies, the proposed solution is also too expert, producing enhanced results of about $90 \%$ accuracy and 94 percent precision. The proposed approach has a wide range of applications since, nowadays, any company needs to make money in the commercial market. Any business hopes that its goods will be well-received in the future. As a result, opinion leaders have a lot of clout in achieving this aim [17]. Using network topology, the impact of opinion leaders or influential people on the collective behavior of a group structure was investigated. The dynamical model employs Axelrod's noninteracting states rules for cultural interaction 
among social agents. Opinion leaders are agents that have a one-way effect over other agents, similar to how a machine is affected by an external industry or the media. Opinion leaders can be as compared to scattered mass media, dispersed advertisers, or a nonuniform marketplace in this context. Based on criteria, 3 combination levels with inside the method had been identified: a managed section wherein opinion leaders set the nation; a nonzero dependent section wherein a huge area is on a nation orthogonal or opportunity to the opinion leaders, casting doubt at the influential hypothesis; and a disordered section wherein a huge area is on a nation orthogonal or opportunity to the opinion leaders, casting doubt at the influential hypothesis; and a disordered section wherein the opinion leaders set the nation. The systems' next mechanism is decided through the scale of the subset of dealers whose states first of all overlap with the ones of the opinion leaders. This explains why, as opposed to sturdy dealers, a vital mass of vulnerable dealers courses such propagation processes. Other nonequilibrium structures with noninteracting states of their dynamics and sufficiently long-variety interactions of their underlying network, in line with our findings, can see the emergence of a self-prepared mechanism with a country aside from leaders. This phenomenon is not an unusual place in social and organic systems that may cluster, aggregate, and migrate and whose dynamics frequently encompass an interplay threshold condition [18].

The evolutionary game theory and simulation paradigm is merged to develop an evolutionary model for agricultural product brands' online promotion strategies that simulate the evolutionary process, validates the model, and investigates the penalty influencing mechanism. The results of a multiagent simulation show that brand marketing pays off regardless of how opinion leaders feel about products. The total advantage strategy employed by agricultural commodity brand companies is discussed. Increased fines for opinion leaders who endorse agricultural product products following negative advertisements can have an effect on their promotion strategies. Furthermore, the proposed research provides an idea and a point of reference for online brand marketing strategies for agricultural product brands [19]. A super network analysis technique was used to classify online public opinion leaders, and a SuperedgeRank algorithm based on the super edge ranking algorithm was proposed. The dominant psychological type, as well as the formation of online public opinion, were introduced. These were examined using psychological models, which may lead to more effective intervention strategies. The mainstream perspective from massive quantities of online posts can be described in the viewpoint subnetwork. If this is a rumor, a quick explanation would be extremely beneficial. Based on the SuperedgeRank algorithm in the super network, numerous studies with different priorities can be performed [20].

It was supposed to use a social network to split opinion leaders into subgroups. Where the target opinions of opinion leader subgroups differed, a very general bounded confidence-based opinion dynamics model for opinion leaders and followers was created. A computer simulation technique was used to investigate the relationship between the proportion of opinion leaders, the support levels of opinion followers, and the levels of confidence in opinion leaders. The studies in e-commerce networks offered a comparative study of a social group's collaborative decisionmaking. According to the comparative study of the three variables, the degree of trust that opinion followers have in opinion leaders has a greater impact on opinion leaders' power ability. As a result, increasing the popularity of opinion leaders is essential for maximizing the e-commerce diffusion effect. Some tools, such as Chukwa, Flume, Scribe, and Kafka, can be used to collect data on social networking sites as community views develop in an e-commerce world [21]. Digital opinion leaders should be used to encourage the experiential (hedonic) and factual appeal of goods and services across a wide range of online platforms. The study looked at how online opinion leaders could be used in a variety of roles, such as appeal leadership, information leadership, and multiple roles. The process of forming alliances with online opinion leaders was broken down into five stages. All steps in the planning process are recognition, alignment, motivation, and rewarding online opinion leaders [22]. The data can play a significant role which need to be analyzed for extracting meaningful information [23].

"Opinion holders" can be detected automatically in BBS in order to identify and direct public opinion in real time. As a result, an algorithm was proposed for detecting "opinion holders" in BBS automatically. Following an assessment of the essential characteristics of "opinion leaders," an X-means iteration clustering filter algorithm based on the Bayesian information value maximization theorem was proposed to locate the opinion leader feature points in the feature space. According to the results of the trial, the model-based algorithm has high precision and recall, meaning that the device can accurately track public opinion. [24]. The replying connections between comments were mapped to the comment writers' relations in an online forum to create a social network. In this dynamic network analysis, network characteristics and computational mechanics techniques were used. Opinion holders in an online community were classified using small-world networks, and their attributes were checked [25]. The role of early decision-making is significant for the successful understanding of business [26-29]. Figure 1 represents the details of the included materials.

\section{Methodology}

In the age of technological advancements, experts may influence acceptance rates by serving as disruptive or powerful opinion leaders. Opinion leaders are individuals in a social network who have the most impact on other people's appreciation or approval of products/services in the process of technological advancement. Opinion leaders, both formally and informally, control the diffusion and acceptance of a new product, as well as other people's choices across various communication networks. As a result, firms' interest in opinion leaders has increased, making opinion leaders a desirable first marketing goal [9]. Because there are so many different types of opinion leaders around the world, 


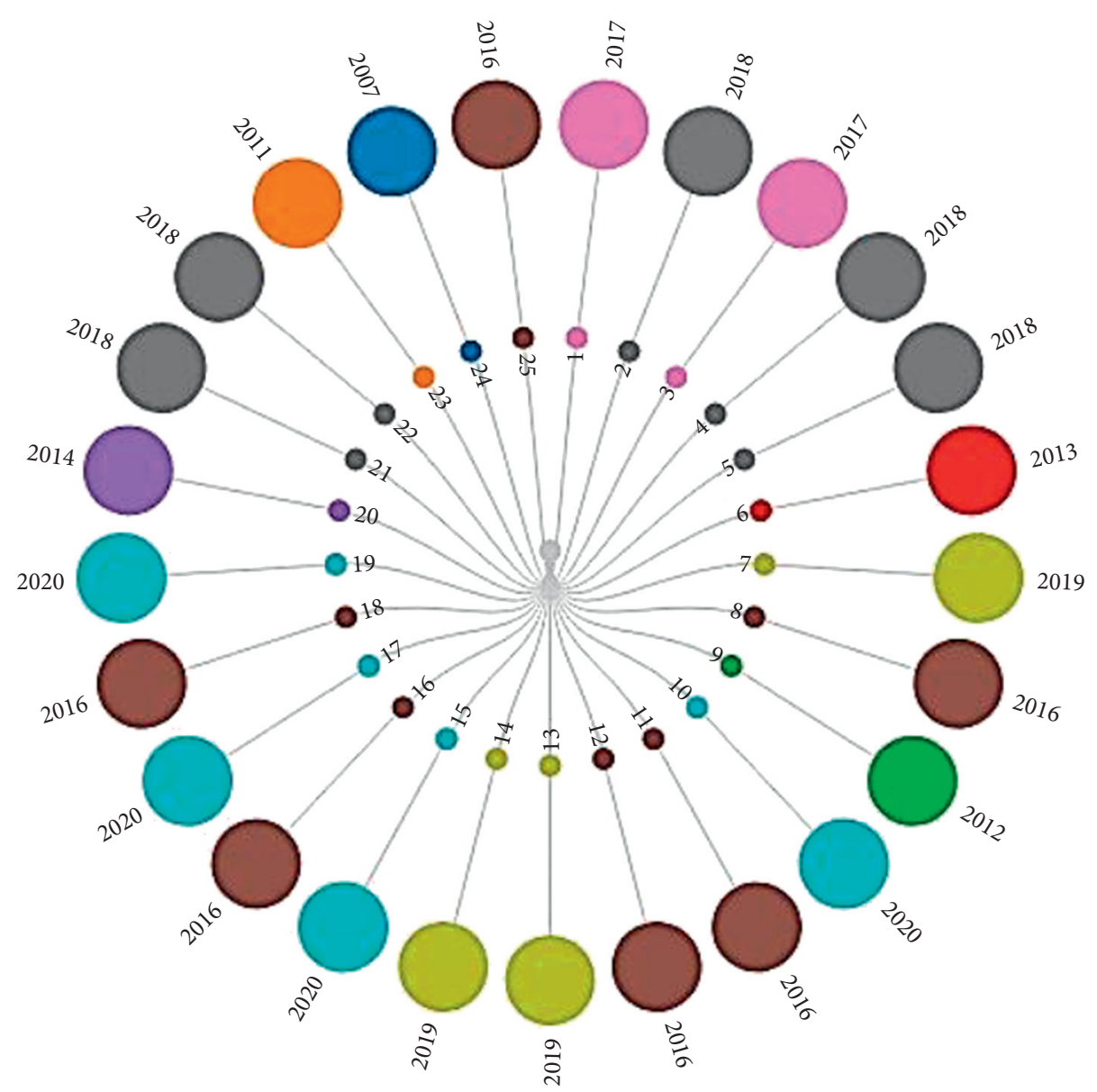

Figure 1: Details of the materials from literature.

choosing the right one can be a difficult task. The selection of an opinion leader will be based on a variety of criteria. These characteristics were discovered through an examination of the literature. Because there are heterogeneous leaders present, the feature set of these leaders will be broad, and there may be redundancy or inappropriate features that influence proper selection. These were filtered to remove inappropriate features.

3.1. Feature-Based Selection. Features selection is carried out to remove inappropriate features to reduce the opinion leader set. As the mixture of features set makes computation difficult, it also causes the dimensionality curse. By eliminating unimportant features, the set is condensed and adequate features are sorted out by utilizing feature-based selection, the features-based selection technique is used to select a subset of the original set while maintaining the original set's precision. Features, efficacy, and scalability can be improved. The selection of suitable leaders is based on their unique features and it is needed when dealing with a wide range of leaders (features) datasets. Due to the profusion of blaring, irrelevant, or deceptive features, the feature-based selection is a necessity in real-world problems. The opinion leaders are heterogeneous in nature, each having its own features. The features of leaders are gathered from previous research studies. It was important to identify these features in order to differentiate between appropriate and inappropriate opinion leaders. The inconsistency and complexity are then removed by filtering these irrelevant features. As a result of reviewing the literature, a set containing various features of opinion leaders is given in Table 1. From the features analyzed during literature analysis, Table 2 is constructed in which two features were united. Our proposed method would pick appropriate opinion leaders for assigning different organizational activities based on these criterial features.

3.2. Ant Colony Optimization. Various approaches have been practiced for diverse issues [31,32]. Ant colony optimization was introduced as a swarm intelligence strategy by M. Dorigo and his colleagues in 1990. [33]. Swarm intelligence is a problem-solving approach focused on animal and insect social experiences. Ants have influenced a wide range of methods and techniques, the most well-known and widely used of which is ant colony optimization, a generalpurpose optimization technique. The foraging behavior of a number of ant species is used to optimize ant colonies. When they (ants) move from one location to another, ants leave pheromones (chemicals) on the ground to mark welcoming directions for other ants in the colony (members). Ant 
TABLe 1: Features of opinion leaders identified from literature.

\begin{tabular}{lcc}
\hline No. & Features & Citation \\
\hline 1 & Observable & {$[4]$} \\
2 & Deep thinker & {$[4]$} \\
3 & Higher competency & {$[4]$} \\
4 & Reputation & {$[4,11,30]$} \\
5 & Active & {$[4]$} \\
6 & Energetic & {$[4]$} \\
7 & Experience & {$[2,4,10,12]$} \\
8 & Knowledge & {$[2,4,10,12]$} \\
9 & Confidence level & {$[7,11,12]$} \\
10 & Authoritative & {$[7]$} \\
11 & Trust & {$[7,10,11]$} \\
12 & Loyal & {$[4]$} \\
13 & Reciprocity & {$[2]$} \\
14 & Expertise & {$[2,30]$} \\
15 & Social capital & {$[2]$} \\
16 & Self-identity & {$[2]$} \\
17 & Decision-makers & {$[10,12]$} \\
18 & Social status & {$[16]$} \\
19 & Education & {$[17]$} \\
20 & Authorized & \\
\hline
\end{tabular}

TABle 2: Opinion leaders set.

\begin{tabular}{lc}
\hline No. & Features \\
\hline O1 & Observable, energetic \\
O2 & Authorized, authoritative \\
O3 & Education, knowledge \\
O4 & Experience, expertise \\
O5 & Self-identity, social capital \\
O6 & Trust, loyal \\
O7 & Confidence, reciprocity \\
O8 & Deep thinker, decision-maker \\
O9 & Active, social status \\
O10 & Competency, reputation \\
\hline
\end{tabular}

colony optimization uses a similar approach to solve optimization problems. To find an optimal solution, the ant colony optimization technique is commonly used [34]. Ants use two variables to solve problems: heuristic knowledge and the frequency of pheromones. Artificial ants that communicate with each other may produce high-quality results. The pheromone trail values are obtained by indirect interaction (sensing the pheromone) between various ants. Ants do not change; instead, they alter how other ants perceive and interpret the dilemma [34]. ACOs may be used to solve a variety of selection complexities.

\subsection{Feature-Based Opinion Leader Selection Using Ant Colony} Optimization. The ACO model can be used to address the issue of identifying and selecting opinion leaders in a society. The proposed framework for feature-based opinion leader selection is implemented using the ant colony optimization technique, and there are several phases in applying the ACO algorithm for opinion leader selection optimization depicted in Figure 2.

The proposed method would address the issue of selecting opinion leaders. The process of selecting a leader starts with the creation of an ant colony, which will travel along different paths (edges) and select a leader on the basis of pheromone value scattered on each edge. If stopping criteria for ant visits are fulfilled, the ants will end (traversal), and the best subset of opinion leaders will be produced, which will then be used in various marketing or industrial settings. If in the visit, the traversal of ants does not meet stopping criteria, the pheromone values are transformed and the loop is restarted.

Opinion leaders present in an ecosystem possess unique features that are identified from existing literature given in Table 1. Opinion leader set $(\mathrm{O})$ is constructed using features identified from the literature given in Table 2. The featurebased opinion leader selection technique will reduce the original set shown in Table 2 by eliminating inappropriate opinion leaders although a higher level of precision in the depiction of the original set will be maintained. As a result, partial selection of opinion leaders takes place. The prior Opinion leader attached to a node does not affect the selection of a potential opinion leader. However, it is not required that the subset formed would be of equal size. The mapping of the opinion leader selection issue into the ACO technique involves the following steps:

(i) Graph structure

(ii) Strength of heuristic function and pheromone

(iii) Pheromone value modification

(iv) Formation of results

3.3.1. Graph Structure. Ant colony optimization usually represents a problem in graphical structure, as shown in Figure 3.

The edges reflect the corresponding opinion leader's decision, while the nodes represent various opinion leaders. The nodes are linked together to allow the selection of any opinion leader. When an ant visits different nodes on the graph, an optimal subset of the opinion leaders is chosen. Stopping requirements must be fulfilled by the ant traversal (select optimal opinion leaders). In Figure 3 , ants $1,2,3,4,5,6,7,8,9$, and 10 are permitted to leave their nest and begin traversing to various nodes such as $\mathrm{O} 1$ or $\mathrm{O} 2$, and then to $\mathrm{O} 3, \mathrm{O} 4, \mathrm{O} 5, \mathrm{O} 6, \mathrm{O} 7, \mathrm{O} 8$, O9, and O10. These ants leave pheromones (a chemical material) on various edges during the traversal process given in Figure 4.

The ants follow the high-level pheromone paths; for example, if pheromone levels are high, the ant will select only high pheromone value edges (bold line) and only those particular nodes. Using the transformation law, ant 1 from the nest will pick node $\mathrm{O} 2$, followed by node $\mathrm{O} 4$. It then selects $\mathrm{O} 6, \mathrm{O}$, and O10. When the ant traversal reaches O10, it hits the stopping criterion and comes to a halt, providing a partial solution to the original opinion leader collection "O," which includes opinion leaders $\mathrm{O} 2, \mathrm{O} 4, \mathrm{O}$, O8, and $\mathrm{O} 10$ as shown in Figure 5. As a consequence, there is a high level of accuracy. The opinion leader subset is then used as a candidate in a variety of industrial, sales, marketing, and influencing environments. 


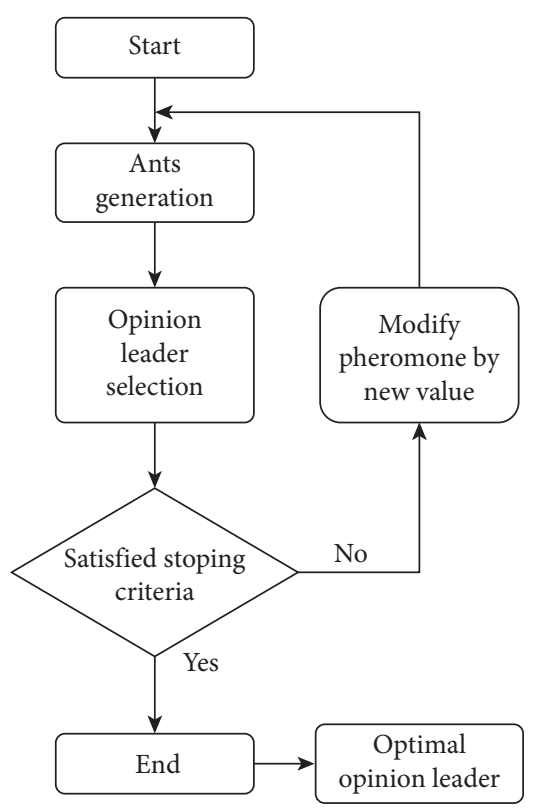

FIGURE 2: Graphical representation of feature-based opinion leader selection using ant colony optimization.

3.3.2. Strength of Heuristic Function and Pheromone. Opinion leaders are evaluated on the basis of optimal features. The initial selection of opinion leader substitutes is made using a simple multistate local search process. In the ACO algorithm, the ( $\eta \mathrm{i})$ heuristic function is used in conjunction with the pheromone value to make a correct transition. Evaluating the pheromone and heuristic meaning yields the best opinion leaders subset. If an opinion leader is selected, the pheromone value that is given to it would be higher. Inappropriate opinion leaders, on the other hand, are rejected based on the pheromone value associated with the respective edge, which is lower. An ant in $\mathrm{O} 1$ decides whether $\mathrm{O} 3$ should be picked or not, and the decision is based on the likelihood of the highest pheromones on the edges, which can be determined using the following formula:

$$
P(\text { Edge })=\frac{P(\text { Pheromones }(X i) \eta i)}{\sum(P(\text { Pheromones }(X i)) \eta i)} .
$$

Equation (1) is used for evaluating ant's probability for selecting a node, probability is represented by $P$, edges or paths are represented by $X i$, and the heuristic intensity is represented by $\eta i$. If an edge is to be chosen, the $\eta$ i value should be held higher; otherwise, it should be lower. The pheromone value influences the traversal and selection of a node (i.e., property). The ant would travel on the edge with the highest pheromone value.

3.3.3. Pheromone Value Modification. If the ant traversal criterion is not met, the pheromone value is changed, new ants are created, and the cycle repeats. Using equation (2), the pheromone value is altered:

$$
t i(t+1)=(1-p) \cdot t i(t)+p \cdot \Delta t i(t),
$$

where $\rho$ is considered as pheromone decay coefficient and the cost assigned to it is in a range of $0-1$. ti is the pheromone amount that is still remaining on a specific path, $\Delta t i$ is considered as pheromone increment for updated iteration process, Best ants leave more pheromones on optimal solution nodes, and as an outcome, optimal opinion leaders are revealed.

3.3.4. Formation of Results. The process for selecting opinion leaders based on features starts with the development of an arbitrary number of artificial ants. The ants are arranged on the graph with their numbers equal to the number of associated opinion leaders. Each ant's traversal begins the process of building a graph from a single (opinion leader) node. Ant travels from a starting point in a probabilistic manner, crossing various nodes before reaching the stopping condition. The resulting set of opinion leaders is gathered and analyzed for an optimal subset. When the best opinion leaders have been found and the findings have been announced, the experiment comes to an end as presented in Figure 5. When the stopping conditions are not met, a pheromone update occurs, a new ant colony is formed, and the process starts over.

\section{Results and Discussion}

Web 2.0 is a social networking site where users can exchange ideas, obtain knowledge, and communicate emotionally on social media sites. People's everyday lives are influenced by social media. Opinion leaders are unique consumers of social media platforms, with higher advanced degrees and a wider following. The views of opinion leaders also affect the public opinion of surrounding users and the entire network. Obviously, opinion leaders are important in social media [5]. Opinion leaders play an important role in influencing public opinion in virtual communities, and it is their dominant status, governance, and social influence that helps them to invite followers [2]. Care must be taken when choosing opinion leaders, as the quality improves 


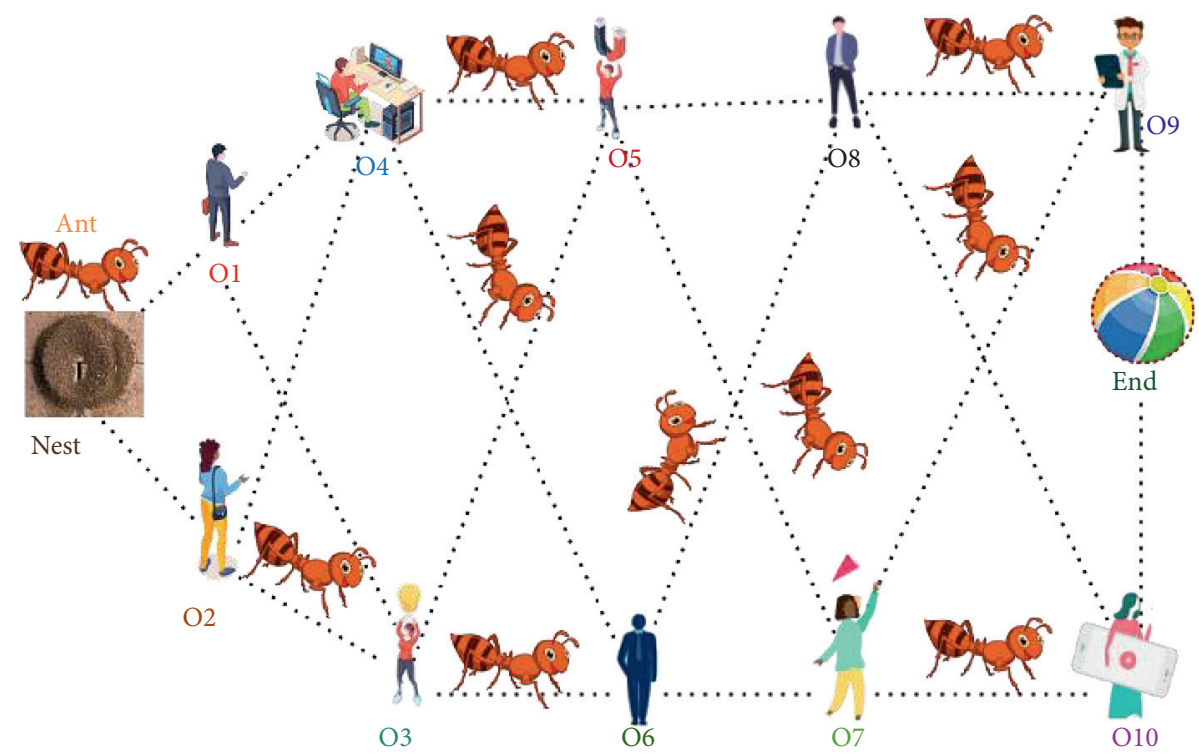

FIgURE 3: Ants traversal on various edges.

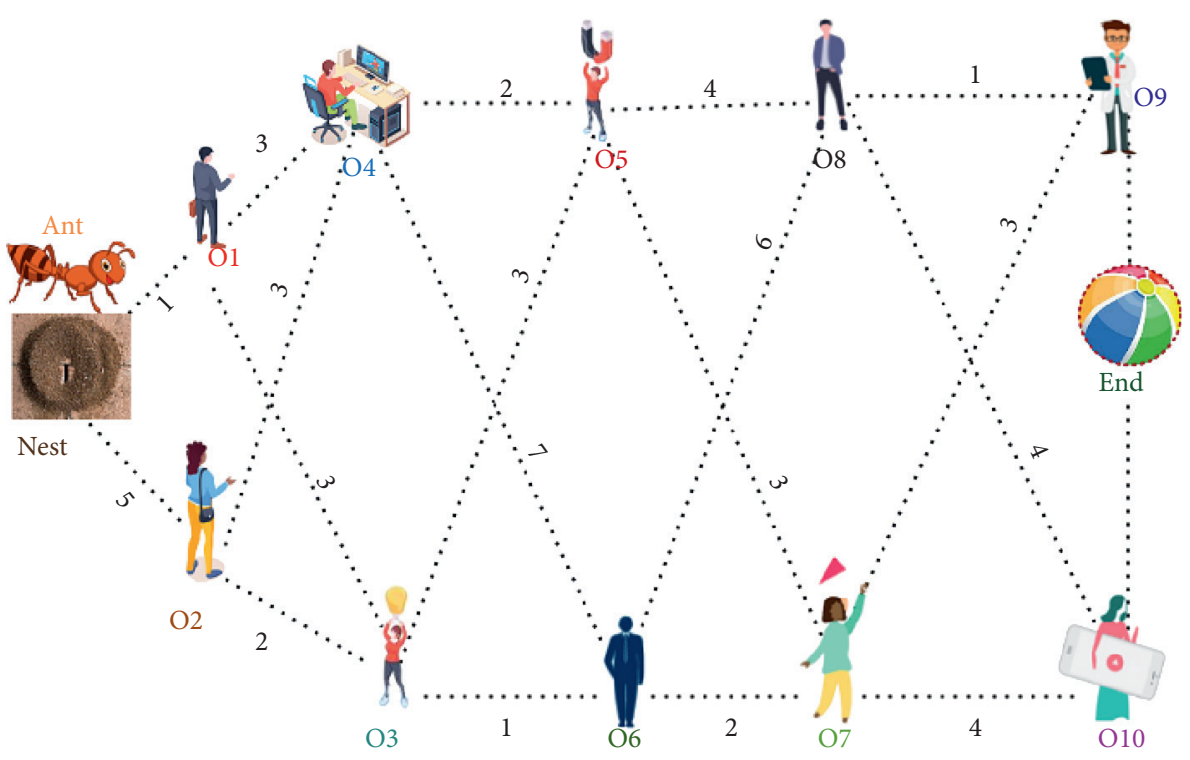

FIgURE 4: Ants excreting pheromones.

with the selection of suitable opinion leaders. An opinion leader set (10 opinion leaders "O1, O2, O3, O4, O5, O6, O7, O8, O9, and O10") with multiple features is placed in a graphical structure in our framework of opinion leader selection based on features. The opinion leaders are portrayed as a collection of nodes linked by edges; an equal number of ants are generated to navigate along multiple edges and pick opinion leaders, resulting in a partial solution of the opinion leader group. If a partial solution meets the stopping criterion, the ants can resist traversing and generate the best opinion leaders subset $\mathrm{O}$ (i.e., it selects the best opinion leaders subset $O$ ). If the ants do not meet the stopping requirements, the pheromones are reset and the process is restarted. The probability of pheromone on each edge decides (opinion leader) selection and rejection; if the value is greater, the nodes are selected, and the best opinion leader's subset is created in ant's traversal; if the value is less, the edges are rejected, and the connected opinion leader node is rejected. Figure 5 depicts the ants and their preferred direction. To find the best traversing path, the likelihood of pheromones on edges is determined, and only suitable opinion leaders are selected. In our proposed opinion leader selection process, we pick the opinion leader's subset $\mathrm{O} 2, \mathrm{O} 4, \mathrm{O} 6, \mathrm{O} 8$, and $\mathrm{O} 10$ by traversing ant 1 because the probability of the values of pheromone on their respective edges connecting the nodes (opinion leaders) was greater than other edges concerning other nodes (opinion leaders). Table 3 shows the selected path by ants. 


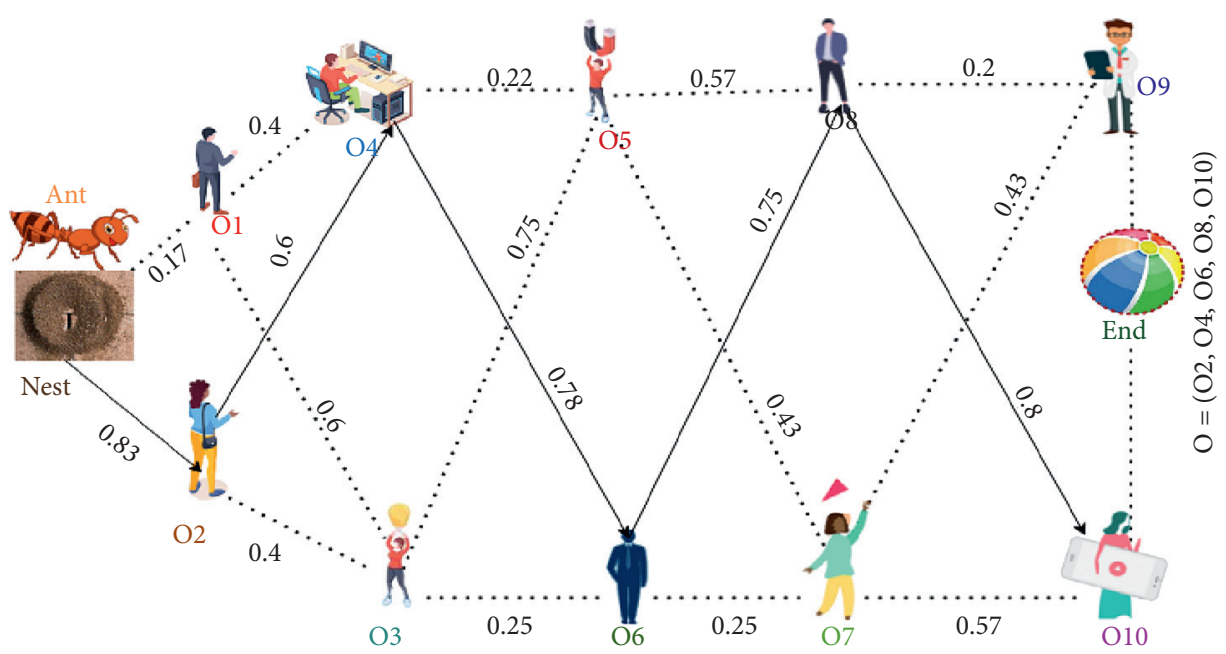

FIgUre 5: Probability wise edges and nodes selection.

TABle 3: Possible paths by ants.

\begin{tabular}{|c|c|c|c|c|c|c|}
\hline $\begin{array}{l}\text { Opinion } \\
\text { leaders }\end{array}$ & $\begin{array}{l}\text { Selected } \\
\text { opinion } \\
\text { leaders }\end{array}$ & Ants & $\begin{array}{l}\text { Pheromone } \\
\text { (value) on } \\
\text { selected edges }\end{array}$ & $\begin{array}{l}\text { Paths selection } \\
\text { probability node } / \Sigma \\
\text { (value) connected } \\
\text { edges }\end{array}$ & $\begin{array}{c}\text { Path selection probability of } \\
\text { an ant (from starting node to } \\
\text { last node) } \sum P \text { (pheromone } \\
\text { on edge) }\end{array}$ & $\begin{array}{c}\text { Probability of pheromone } \mathrm{P} \\
\text { (pheromone on selected } \\
\text { edges) } / \sum P \text { (pheromones on } \\
\text { all edges) }\end{array}$ \\
\hline \multirow{11}{*}{$\begin{array}{l}\mathrm{O} 1, \mathrm{O} 2, \mathrm{O} 3 \\
\mathrm{O} 4, \mathrm{O} 5, \mathrm{O} 6 \\
\mathrm{O} 7, \mathrm{O} 8, \mathrm{O} 9 \\
\mathrm{O} 10\end{array}$} & $\begin{array}{l}\mathrm{O} 2, \mathrm{O} 4 \\
\mathrm{O} 6, \mathrm{O} 8 \\
\mathrm{O} 10\end{array}$ & 1 & $5,3,7,6,4$ & $\begin{array}{c}0.83,0.6,0.78,0.75 \\
0.8\end{array}$ & 3.76 & 0.15 \\
\hline & $\begin{array}{c}\mathrm{O} 1, \mathrm{O} 4 \\
\mathrm{O} 5, \mathrm{O} 8, \mathrm{O} 9\end{array}$ & 2 & $1,2,2,4,1$ & $\begin{array}{c}0.17,0.4,0.22,0.5 \\
0.2\end{array}$ & 1.56 & 0.06 \\
\hline & $\begin{array}{c}\mathrm{O} 2, \mathrm{O} 3, \\
\mathrm{O} 5, \mathrm{O} 8, \mathrm{O} 9\end{array}$ & 3 & $5,2,3,4,1$ & $\begin{array}{c}0.83,0.4,0.75,0.57 \\
0.2\end{array}$ & 2.75 & 0.11 \\
\hline & $\begin{array}{l}\mathrm{O} 1, \mathrm{O} 3 \\
\mathrm{O} 6, \mathrm{O} 7 \\
\mathrm{O} 10\end{array}$ & 4 & $1,3,1,2,4$ & $\begin{array}{c}0.17,0.6,0.25,0.25 \\
0.57\end{array}$ & 1.84 & 0.08 \\
\hline & $\begin{array}{c}\mathrm{O} 2, \mathrm{O} 3, \\
\mathrm{O} 6, \mathrm{O} 8, \mathrm{O} 9\end{array}$ & 5 & $5,2,1,6,1$ & $\begin{array}{c}0.83,0.4,0.25,0.75 \\
0.2\end{array}$ & 2.43 & 0.10 \\
\hline & $\begin{array}{c}\mathrm{O} 2, \mathrm{O} 3, \\
\mathrm{O} 6, \mathrm{O} 7, \mathrm{O} 9\end{array}$ & 6 & $5,2,1,2,3$ & $\begin{array}{c}0.83,0.4,0.25,0.25 \\
0.43\end{array}$ & 2.16 & 0.09 \\
\hline & $\begin{array}{l}\mathrm{O} 1, \mathrm{O} 4 \\
\mathrm{O} 6, \mathrm{O} 8 \\
\mathrm{O} 10\end{array}$ & 7 & $1,2,7,6,4$ & $\begin{array}{c}0.17,0.4,0.78,0.75 \\
0.8\end{array}$ & 2.9 & 0.12 \\
\hline & $\begin{array}{c}\mathrm{O} 1, \mathrm{O} 3, \\
\mathrm{O} 5, \mathrm{O} 7, \mathrm{O} 9\end{array}$ & 8 & $1,3,3,3,3$ & $\begin{array}{c}0.17,0.6,0.75,0.43 \\
0.43\end{array}$ & 2.38 & 0.10 \\
\hline & $\begin{array}{c}\mathrm{O} 1, \mathrm{O} 4 \\
\mathrm{O} 5, \mathrm{O} 7, \mathrm{O} 9\end{array}$ & 9 & $1,2,2,3,3$ & $\begin{array}{c}0.17,0.4,0.22,0.43 \\
0.43\end{array}$ & 1.65 & 0.07 \\
\hline & $\begin{array}{l}\mathrm{O} 2, \mathrm{O} 3 \\
\mathrm{O} 6, \mathrm{O} 8 \\
\mathrm{O} 10\end{array}$ & 10 & $5,2,1,6,4$ & $\begin{array}{c}0.83,0.4,0.25,0.75 \\
0.8\end{array}$ & 3.03 & 0.12 \\
\hline & & & & & $\sum=24.46$ & \\
\hline
\end{tabular}

\section{Conclusion}

Web 2.0 has become increasingly important in people's daily lives. Many online communities, such as forums, microblogs, and websites, are becoming more interactive. People can quickly express themselves and read what others have to say. An influential leader who is an expert in a specific field and has a large number of people who follow his or her opinions or thoughts on social media is known as an opinion leader. Without a question, opinion leaders are the thinking drivers and message senders who are familiar with the media by secondary transmission. The identification of opinion leaders in a social network has important economic and political consequences. Businesses and governments will contact the most influential people after discovering them in order to manipulate revenues or guide public sentiment, respectively. To date, several models have been proposed to investigate the mechanisms of leaders. However, defining opinion leaders in the network is difficult due to the social network's high complexity and the randomness and 
contingency of their behavior. Our study also focuses on the issue of selecting opinion leaders based on their distinct characteristics. Feature-based opinion leader selection using ant colony optimization for the opinion leader selection problem was proposed which selects appropriate opinion leaders. Our research makes a significant contribution by assisting in the selection of a suitable opinion leader based on the criterial features. Choosing the right opinion leader would improve the productivity and effectiveness of different organizations. This article potentially discusses the method with fewer opinion leaders features. We will use it again because it is effective (i.e., it selects opinion leaders based on criterial features) and will play an important role in the selection of opinion leaders in any context.

\section{Data Availability}

No data were used to support this study.

\section{Conflicts of Interest}

The authors declare that they have no conflicts of interest regarding the publication of this paper.

\section{References}

[1] F. Wang, "An empirical study of opinion leader in online social network," in Proceedings of the 2017 3rd IEEE International Conference on Computer and Communications (ICCC), pp. 1493-1497, IEEE, Chengdu, China, December 2017.

[2] Y. Xiong, Z. Cheng, E. Liang, and Y. Wu, "Accumulation mechanism of opinion leaders' social interaction ties in virtual communities: empirical evidence from China," Computers in Human Behavior, vol. 82, pp. 81-93, 2018.

[3] Y.-C. Chen, Y.-H. Chen, C.-H. Hsu, H.-J. You, J. Liu, and $\mathrm{X}$. Huang, "Mining opinion leaders in big social network," in Proceedings of the 2017 IEEE 31st International Conference on Advanced Information Networking and Applications (AINA), pp. 1012-1018, IEEE, Taipei, Taiwan, March 2017.

[4] L. Jain and R. Katarya, "A systematic survey of opinion leader in online social network," in Proceedings of the 2018 International Conference on Soft-computing and Network Security (ICSNS), pp. 1-5, IEEE, Coimbatore, India, February 2018.

[5] H. Cao, J. Wang, and Z. Wang, "Opinion leaders discovery in social networking site based on the theory of propagation probability," in Proceedings of the 2018 2nd IEEE Advanced Information Management, Communicates, Electronic and Automation Control Conference (IMCEC), pp. 700-704, IEEE, Xi'an, China, May 2018.

[6] Z. Wei and H. Ming-sheng, "Influence of opinion leaders on dynamics and diffusion of network public opinion," in Proceedings of the 2013 International Conference on Management Science and Engineering 20th Annual Conference Proceedings, pp. 139-144, IEEE, Harbin, China, July 2013.

[7] Y. Lu, Y. Zhao, J. Zhang, J. Hu, and X. Hu, "Fuzzy hegselmann-krause opinion dynamics with opinion leaders," in Proceedings of the 2019 Chinese Control Conference (CCC), pp. 6019-6024, IEEE, Guangzhou, China, July 2019.

[8] Y. Zhao and G. Kou, "Opinion evolution of a social group with extreme opinion leaders," in Proceedings of the 2016 6th International Conference on Computers Communications and
Control (ICCCC), pp. 70-74, IEEE, Oradea, Romania, May 2016.

[9] Y. Cho, J. Hwang, and D. Lee, "Identification of effective opinion leaders in the diffusion of technological innovation: a social network approach," Technological Forecasting and Social Change, vol. 79, no. 1, pp. 97-106, 2012.

[10] P. Gao, J. Hung, and Y. Xu, "A k-core decomposition-based opinion leaders identifying method and clustering-based consensus model for large-scale group decision making," Computers \& Industrial Engineering, vol. 150, Article ID 106842, 2020.

[11] M. Chiregi and N. J. Navimipour, "A new method for trust and reputation evaluation in the cloud environments using the recommendations of opinion leaders' entities and removing the effect of troll entities," Computers in Human Behavior, vol. 60, pp. 280-292, 2016.

[12] Y. Zhao, L. Zhang, M. Tang, and G. Kou, "Bounded confidence opinion dynamics with opinion leaders and environmental noises," Computers \& Operations Research, vol. 74, pp. 205-213, 2016.

[13] S. A. Semenkovich and O. A. Tsukanova, "On the algorithms of identifying opinion leaders in social networks," Procedia Computer Science, vol. 162, pp. 778-785, 2019.

[14] C. Li, J. Bai, L. Zhang, H. Tang, and Y. Luo, "Opinion community detection and opinion leader detection based on text information and network topology in cloud environment," Information Sciences, vol. 504, pp. 61-83, 2019.

[15] L. Jain, R. Katarya, and S. Sachdeva, "Opinion leader detection using whale optimization algorithm in online social network," Expert Systems with Applications, vol. 142, Article ID 113016, 2020.

[16] S. M. Aghdam and N. Jafari Navimipour, "Opinion leaders selection in the social networks based on trust relationships propagation," Karbala International Journal of Modern Science, vol. 2, no. 2, pp. 88-97, 2016.

[17] L. Jain, R. Katarya, and S. Sachdeva, "Recognition of opinion leaders coalitions in online social network using game theory," Knowledge-Based Systems, vol. 203, Article ID 106158, 2020.

[18] K. Tucci, J. C. González-Avella, and M. G. Cosenza, "Rise of an alternative majority against opinion leaders," Physica A: Statistical Mechanics and Its Applications, vol. 446, pp. 75-81, 2016.

[19] M. Liao, J. Zhang, R. Wang, and L. Qi, "Simulation research on online marketing strategies of branded agricultural products based on the difference in opinion leader attitudes," Information Processing in Agriculture, 2020.

[20] N. Ma and Y. Liu, "SuperedgeRank algorithm and its application in identifying opinion leader of online public opinion supernetwork," Expert Systems with Applications, vol. 41, no. 4, pp. 1357-1368, 2014.

[21] Y. Zhao, G. Kou, Y. Peng, and Y. Chen, "Understanding influence power of opinion leaders in e-commerce networks: an opinion dynamics theory perspective," Information Sciences, vol. 426, pp. 131-147, 2018.

[22] H.-C. Lin, P. F. Bruning, and H. Swarna, "Using online opinion leaders to promote the hedonic and utilitarian value of products and services," Business Horizons, vol. 61, no. 3, pp. 431-442, 2018.

[23] Y. Huang, Z. Xiao, D. Wang, H. Jiang, and D. Wu, "Exploring individual travel patterns across private car trajectory data," IEEE Transactions on Intelligent Transportation Systems, vol. 21, no. 12, pp. 5036-5050, 2019. 
[24] S. Zhu, X.-l. Zheng, and D. Chen, "Research of algorithm for automatic opinion leader detection in BBS," System Engineering Theory and Practice, vol. 31, pp. 7-12, 2011.

[25] J. Gao and J. Yang, "Analysis of opinion leader in on-line communities," Journal of University of Electronic Science and Technology of China, vol. 36, no. 6, p. 1249, 2007.

[26] J. Zhang, S. Nazir, A. Huang, and A. Alharbi, "Multicriteria decision and machine learning algorithms for component security evaluation: library-based overview," Security and Communication Networks, vol. 2020, Article ID 8886877, 14 pages, 2020.

[27] H. Xiaolong, Z. Huiqi, Z. Lunchao, S. Nazir, D. Jun, and A. S. Khan, "Soft computing and decision support system for software process improvement- a systematic literature review," Scientific Programming, vol. 2021, Article ID 7295627, 2021.

[28] Q. Hao, S. Nazir, X. Gao, L. Ma, and M. Ilyas, "A review on multicriteria decision support system and industrial internet of things for source code transformation," Scientific Programming, vol. 2021, Article ID 6661272, 9 pages, 2021.

[29] J. Li, A. Ullah, J. Li et al., "Attributes-based decision making for selection of requirement elicitation techniques using the analytic network process," Mathematical Problems in Engineering, vol. 2020, Article ID 2156023, 13 pages, 2020.

[30] S. Chen, D. H. Glass, and M. McCartney, "Characteristics of successful opinion leaders in a bounded confidence model," Physica A: Statistical Mechanics and Its Applications, vol. 449, pp. 426-436, 2016.

[31] T. A. A. Ali, Z. Xiao, J. Sun, S. Mirjalili, V. Havyarimana, and H. Jiang, "Optimal design of IIR wideband digital differentiators and integrators using salp swarm algorithm," Knowledge-Based Systems, vol. 182, Article ID 104834, 2019.

[32] Z. Xiang, Z. Xiao, D. Wang, and H. M. Georges, "Incremental semi-supervised kernel construction with self-organizing incremental neural network and application in intrusion detection," Journal of Intelligent and Fuzzy Systems, vol. 31, no. 2, pp. 815-823, 2016.

[33] M. Dorigo and C. Blum, "Ant colony optimization theory: a survey," Theoretical Computer Science, vol. 344, no. 2-3, pp. 243-278, 2005.

[34] M. Dorigo, M. Birattari, and T. Stutzle, "Ant colony optimization," IEEE Computational Intelligence Magazine, vol. 1, no. 4, pp. 28-39, 2006. 